\title{
Feedback
}

\section{The complete self-management information system}

In HSM 5 (Spring 1985) in the overview of my paper 'Information systems for self-management', the comments, while generally positive, misinterpret some key points of the paper. Since errors have also been made by other readers, I would like to restate some points more clearly.

While it is true that I argue that certain judgemental functions performed by humans could be replaced by computers, these functions do not actually require judgment. For instance, distribution of agendas to all persons who have certain key words in a search profile, or calculation of vote distributions in complicated voting schemes. In bureaucratically mediated organizations judgments are often exercised in these cases in the name of expediency, often at the expense of fairness to the participants. The point of the article is that human judgment should be exercised only by those affected by those judgments, to as great a degree as possible.

Second, it makes no difference whether individuals' search profiles are stored in a central computer or in personal computers. All notices are 'broadcast' to all users. This can take place inside a computer, through a machine-readable 'newspaper of record', or even over the subcarrier of an FM radio station. Security and privacy considerations might make one of these alternatives superior to another, but such questions are not addressed in the article. The viewpoint of the article is the 'systems' view which could mislead the reader into thinking that central control was an important feature, when in fact it is merely incidental to the exposition.

From a user's view, the system could be best explained as based upon a personal computer

North-Holland

Human Systems Management 5 (1985) 261-262 package which might be called 'The complete self-management information system'.

It would perform the following functions:

(1) Information retrieval. Scan the 'newspaper of record' for items of interest to the user. Retrospective searches of document and statistical data bases would be supported to assist in 2 .

(2) Facilitate participation. Assist in preparation and secure transmission of position papers, agenda items, and votes according to rules previously selected. Scheduling of meetings and calendar management would be included in this participation function.

(3) Feedback collection. Retrieve results of each transmission, verifying reception, scheduling arguments for rebuttal, and transferring cash vouchers through to an Electronic Funds Transfer system for credit. Accounting functions would be performed by this module. These results could be used to automatically alter the operations 1 and 2 above according to a predetermined optimization strategy.

(4) Guaranteeing individual rights. Verify that all actions above followed the constitutional principles and bylaws of the organization, including conforming to accepted accounting principles.

(5) Adjudication of disputes. Submit a complaint for judicial review if violations are noted in 4 . The system would have facilities to organize all aspects of a judicial proceeding.

(6) Focus awareness. Randomly select items from the 'newspaper of record' to insure monitoring of all activities and to expand awareness of possibilities for involvement in the organization. (These could also be randomly selected if desired.)

It should be clear from this description that 'judgment' can be mechanized to a degree desired 
by each individual (to the limits of the state of the art) and that this is no way inhibits a person from viewing each item. The point of the article is the maximization of self-determination in the face of great complexity.

The statement that 'Democracy has absolutely no mechanism to insure quality of performance in the voting process', is not correct except in its narrowest sense. The protection of minorities, the airing of opposing views according to rules of procedure, and protection of expression by the secret ballot are all ways of insuring the quality of performance. The greatest insurance is the learning process which results when people see the effect of their decisions. This, of course, goes beyond the voting process itself, to include the other elements mentioned above.

The statement 'A majority can be wrong, and actually must be wrong, more often than minorities or individuals; ...' is also only correct in a narrow sense. In general, increasing numbers, in a democratic system, tend toward more correct decisions. Of course, some minority will often be right, but which one?

Finally, the concluding portion of the previous quote, '... the one thing it does assure is that the deviations from the average opinion or view will be minimized by increased participation', seems to contradict the diversity which is promoted by the protection of a democratic system. It is true in the sense that in a truly democratic system all information is shared, thereby reducing deviation from the average opinion (and in the limit eliminating voting, since a concensus is reached. This can hardly be seen as a disadvantage).

David STODOLSKY

Institute for Organization and Management Aarhus School of Business Administration DK-8210 Aarhus, V, Denmark 